\title{
Effect of Segment Length on Domain Wall Pinning in Multisegmented Co/Ni Nanowires for 3D Memory Applications
}

\author{
Julián A. Moreno ${ }^{1,1, *}$, Hanan Mohammed ${ }^{2}$, Jürgen Kosel ${ }^{2}$
}

\begin{abstract}
The interfaces between different materials in multisegmented nanowires act as pinning centers for domain walls, making these nanowires attractive materials for 3D memory devices. Here, the switching events which accompany a domain wall pinning and depinning in two-segmented $\mathrm{Co} / \mathrm{Ni}$ nanowires with $80 \mathrm{~nm}$ in diameter have been simulated for various segment lengths, using the MAGPAR package within the Virtual Micromagnetics environment. Different switching mechanisms of the magnetization were found for nanowires with different segment lengths, contributing to different values of the pinning and depinnning fields. Domain wall pinning is caused by the stray field from the Co segment; therefore, the position of the pinned domain wall depends on the cobalt segments length: in case of the smaller segment lengths, the domain wall is pinned at the interface itself, whereas in case of $700 \mathrm{~nm}$ segments a $150 \mathrm{~nm}$ displacement of the pinned domain wall from the interface is found, consistent with experimental reports. Domain wall pinning is manifested as a plateau in the magnetization curve. In case of nanowires with shorter segments, another plateau is observed that is related to the creation of a magnetic vortex structure. These findings are crucial towards determining the minimum segment length to achieve a higher
\end{abstract}

\footnotetext{
${ }^{*}$ Corresponding author

${ }^{1}$ King Abdullah University of Science and Technology (KAUST), Physical Science and Engineering Division (PSE), Thuwal 23955-6900, Saudi Arabia

${ }^{2}$ King Abdullah University of Science and Technology (KAUST),

Computer, Electrical and Mathematical Science and Engineering Division (CEMSE), Thuwal 23955-6900, Saudi Arabia
}

Preprint submitted to Journal of Magnetism and Magnetic Materials (JMMM)February 3, 2019 
bit density that displays optimal pinning and depinning fields.

Keywords: Micromagnetic Simulation, Cylindrical Nanowire, Domain Wall, Magnetic Memory, Nanofabrication, Domain Wall Motion

\section{Introduction}

The quest for next generation devices is pushing the boundaries of various technologies such as logic and data storage devices. The racetrack-memory concept [1] based on cylindrical nanowires (CNW) 2] shows considerable po-

5 tential as a next-generation data storage device. In this memory, multiple bits or magnetic domains can be incorporated in a single CNW by tuning various parameters such as diameter [3] or chemical composition [4, 5]. Experimental studies have shown that in chemically modulated Co/Ni CNWs, the pinning of domain walls occurs near the interface, due to the larger stray field emanating from the cobalt segments [6, 7, 8, which can be exploited to create and manipulate 9 multi-bit states along such a CNW. The concept of such a memory depends on the readout method. In case of magnetoresistance measurements 4], a magnetization along the $\mathrm{CNW}$ axis results in the same resistance value, independent of the direction. Hence, two segments are required for each bit. In these studies, the segment length is a consequence of fabrication and characterization requirements, and the fact that above a certain aspect ratio, the stray field strength saturates. For practical purposes, this suggests an upper limit of segment length, but there is no similar criteria for a lower limit.

In this paper we use micromagnetic simulations to systematically study the effect of the segment length on the pinning and depinnning of a domain wall at the interface of the nickel and cobalt segments. The domain wall pinning and depinning fields are studied, as well as their absolute difference, defined as a depinning "strength". 


\section{Materials and methods}

25 Micromagnetics environment 11 was used to search for the ground state of a two-cylinder magnetic bodies system at different values of the applied field. Perfect interfaces were assumed, i.e. sharp, flat and no materials mixing. This is commonly known as finding the relaxed states of the system. The effect of was considered to be negligible, due to its short range (few nanometers at most) compared to the large contributions of crystal and shape anisotropies. This means that the interfaces are sharp: the gradient in composition was considered to be below or in the order of the element size, which is commonly found experwith the $\mathrm{z}$ axis in Cartesian coordinates, and, as initial state, the magnetization of the two bodies was artificially set to the positive $\mathrm{z}$ direction $\left(m_{z}=1\right)$ and was allowed to relax without an external field. Due to the large shape anisotropy arising from the aspect ratio, most of the $\mathrm{z}$ magnetization component remains

40 in that direction in the relaxed state. After this, an external magnetic field was applied in the negative $\mathrm{z}$ direction, to reverse the magnetization of the bodies and allow the system to relax again. The external field was applied 1 degree away from the wire's axis and increased in steps of 10 Oe. The simulation was conditioned to stop, when the magnetization of the bodies reached $m_{z} \leq-0.95$.

\subsection{Simulation parameters}

The tetrahedral mesh was generated with linear elements of edge length in the order of the exchange length of cobalt. This is smaller than the smallest expected feature, which is normally the domain wall width, and big enough to meet computing memory and time demands. In the case of CNWs of about 100 $\mathrm{nm}$ in diameter, the domain wall width is in the order of $100 \mathrm{~nm}$ [12, 6]. In our simulations, the memory requirements limited the element size to an average length of $4.6 \mathrm{~nm}$, which is more than enough to resolve the domain wall width. 


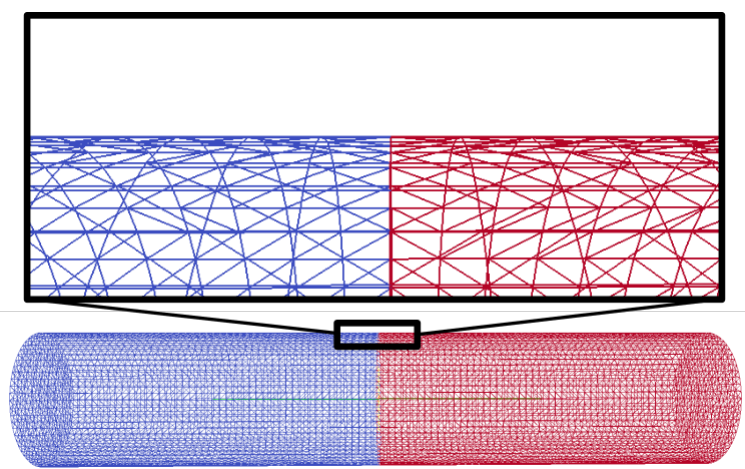

Figure 1: The finite element model of a two-segmented cylindrical nanowire is made of two perfect magnetic cylinders with a shared boundary (interface) between them. The magnetic domains are shown in different colors (red and blue).

Table 1: Parameters used in the simulations.

\begin{tabular}{ccccc}
\hline Material & $M_{s}(T)$ & $A(\mathrm{~J} / \mathrm{m})$ & $K_{1}\left(\mathrm{~J} / \mathrm{m}^{3}\right)$ & $K_{2}\left(\mathrm{~J} / \mathrm{m}^{3}\right)$ \\
\hline Nickel & 0.61 & $3.4111 \times 10^{-12}$ & $-0.048 \times 10^{5}$ & 0.0 \\
Cobalt & 1.75 & $1.32698 \times 10^{-11}$ & $-2.7 \times 10^{5}$ & $-0.75 \times 10^{5}$ \\
\hline
\end{tabular}

The boundary (interface) between the cylinders is shared, as seen in Fig. 1 . which shows the domains of the different magnetic materials in different colors 55 (red and blue).

The magnetic parameters considered in the simulations are summarized in Table 1 and are taken from [13] for fcc Ni (111) and Co (111). Where $M_{s}(T)$ is the saturation magnetization, $A(J / m)$ is the exchange constant and $K_{i}\left(\mathrm{~J} / \mathrm{m}^{3}\right)$ are the magnetocrystalline anisotropy constants. The latter define an energy surface, which was oriented (rotated) using the Euler angles $\theta=0.9, \phi=0.0, \psi=0.61 \mathrm{rad}[14$. 


\section{Results and Discussions}

\subsection{Characteristic Fields}

Fig. 2a shows the $\mathrm{z}$ component of the magnetization starting from remanence 65 in the positive $\mathrm{z}$-direction, when a field is applied in the negative $\mathrm{z}$-direction, for different segment lengths. In general, the magnetization reversal depends on the segment length and a complex interplay of shape anisotropy, crystal anisotropy (dominant in the Co segment) and the effect of the stray field of the Co segment inside of the $\mathrm{Ni}$ segment. The magnetization inside of the Ni segment usually switches its direction before the one in the Co segment, due to the smaller exchange and crystal anisotropy energies in the $\mathrm{Ni}$ segment. With the exception of the $50 \mathrm{~nm}$ long segments, as the field increases, the $\mathrm{z}$ component of the magnetization slowly reduces, due to open vortices at the ends of the CNW, which grow in longitudinal size and are shown for $700 \mathrm{~nm}$

75 long segments in Fig. 2b for the states (i) and (ii) in Fig. 2a (the magnetization in the positive $\mathrm{z}$ direction is red and the magnetization in the negative $\mathrm{z}$ direction is blue). This image shows a transversal cut along the center of the nanowire and the respective end views of each state. As seen in state (ii) from figure $2 \mathrm{~b}$, these open vortices extend around $390 \mathrm{~nm}$ from the end, and exhibit the 80 "vortex tube" structure reported in other works [15], yet they do not display the Bloch Skyrmion tube configuration also reported there. After state (ii) a sudden drop of the magnetization is observed in which, in case of the CNW with $700 \mathrm{~nm}$ long segments, the open vortex on the $\mathrm{Ni}$ end transforms into a domain wall and moves from the end to the interface, as has been commonly found for long nanowires [16]. This results in switching the magnetization direction in the Ni segment. A plateau with almost constant magnetization follows, where the domain wall remains pinned at the interface (Fig. 2b (iii)) until another sudden drop in magnetization occurs, which is caused by the depinning and movement of the domain wall (Fig. 2b (iv)), switching the magnetization in the

90 Co segment. In case of 600 and $700 \mathrm{~nm}$ long segments, only one such plateau can be observed between two switching events. This kind of magnetization reversal 
mechanism applies to nanowires with relatively long segments, i.e. longer than $600 \mathrm{~nm}$. The field at which only the nickel segment is switched defines a pinning field and the consecutive switching of the cobalt segment defines a depinning field. The absolute value of the difference of these two fields is defined as a "depinning strength".

For $400 \mathrm{~nm}$ and $500 \mathrm{~nm}$ segment length no pinning was observed even after reducing the field step to 5 Oe. Instead of a domain wall nucleation, movement and pinning, the open vortex grows in length towards the interface, and at the value of the switching field, the magnetization in both segments switches. We attribute the fact that no domain wall was nucleated in those cases to the shorter lengths of the segments, leading to less shape anisotropy effects and a coupling with the stray field from the Co segment. If a domain wall pinning exists, it is very unreliable within less than 5 Oe, which is technologically irrelevant in our

In case of the 100, 200 and $300 \mathrm{~nm}$ long segments, the open vortex in the $\mathrm{Ni}$ segment continues growing from the CNW's end toward the interface, creating a core with magnetization pointing along the $\mathrm{z}$ axis and a shell with magnetization in circumferential direction (state (ii) in Fig. 2a and Fig S1). Upon reaching the value of the switching field, the magnetization in the $\mathrm{Ni}$ segment switches by coherent rotation and a domain wall appears at the interface.

Notice here that this open vortex reaches the interface before it transforms into a domain wall, that is, the segment length is shorter than the maximum possible open vortex's extension found for 600 and $700 \mathrm{~nm}$ segment lengths, hence the difference in switching mechanisms. This is followed by a plateau during which the domain wall stays pinned at the interface. During this state, the short Co segment has a vortex-like magnetic configuration with the core magnetized along the $\mathrm{z}$ axis and the shell rotating about it. After depinning the domain, a jump to another plateau switches only this core in the Co segment. ${ }_{120}$ At the end of this second plateau, the shells magnetization also rotates into the z-direction $\left(m_{x}\right.$ and $\left.m_{y}\right)$ (See supplemental movie). In case of the $300 \mathrm{~nm}$ long segments, the first plateau is caused by a change in the magnetization 
configuration of both segments to a vortex structure. The second plateau arises after switching the $\mathrm{Ni}$ segment at which point a domain wall is pinned at the interface. Finally, the Co segment is switched and the value of the magnetization jumps to the saturation value. We attribute this reconfiguration of the out of axis magnetization (magnetic vortex structure) observed for lower aspect ratio CNWs to the reduced shape anisotropy energy in comparison to the high aspect ratio CNWs with the same crystal anisotropy energy. For further details on the magnetization processes see supplementary movie M1.

The field values for pinning, depinning and depinning strength are plotted in Fig. 3. For $300 \mathrm{~nm}$ and below, the increasing depinning field as the segment length is reduced can be understood from an effective field perspective. Aside from the applied in-axis field, crystal and shape anisotropy contribute to the total field. The crystal anisotropy energy surface, favors the magnetization with out of axis components (see section 2.1), acting as an out-of-axis field. The shape anisotropy, on the other hand, depending on the segment length acts as an in-axis (long segments) or out-of-axis (short segments) effective field. So, for longer segments, the contribution from the in-axis field from the nickel segment increases. Therefore, the effective in-axis field acting on the domain wall is higher for longer segments.

The impact of the shape anisotropy effective field can be better appreciated at remanence (zero applied field) in Fig. 2a). As the segment length shortens, the in-axis magnetization $\left(m_{z}\right)$ decreases. In this case, instead of acting on a domain wall, the effective out-of-axis field helps nucleate vortexes at the ends which switch the nickel segment followed by pinning of the domain wall. As the segment length increases, the in-axis effective field opposes the applied field and so, more field is needed to pin the domain wall.

In case of the 600 and $700 \mathrm{~nm}$ segment length CNW, the switching mechanism is different, i.e., in this case, there is a propagation of a domain wall from the depinning (end of plateau in Fig. 2a) into the cobalt, for which a lower field is needed, whereas in the $300 \mathrm{~nm}$ or less segment lengths, the quasi coherent reversal of the core in the cobalt segment is required (See Fig. 4 and 


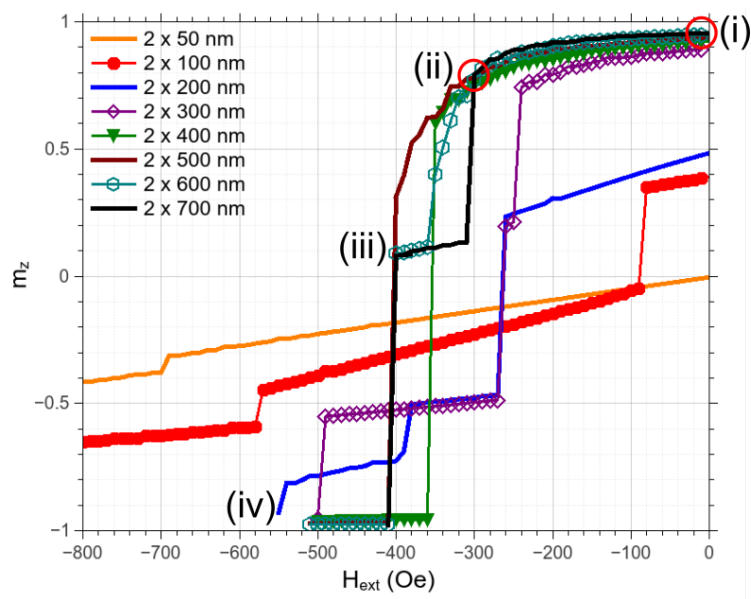

(a) Magnetization along the nanowire axis $\left(m_{z}\right)$ as function of the applied field $\left(H_{\text {ext }}\right)$ and segment length: Remanence (i) first switching event (ii) plateau (pinned domain wall)

(iii) and second switching event (iv).

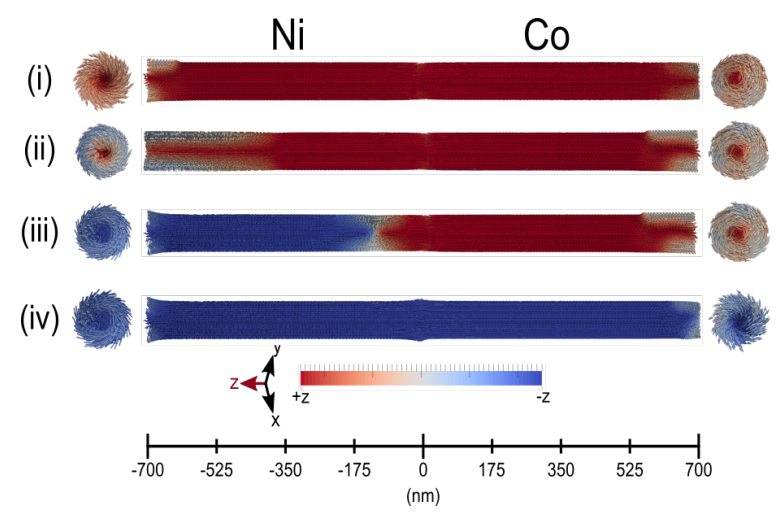

(b) Magnetization profile of a CNW with $700 \mathrm{~nm}$ long segments sliced in half through the long axis at the different stages labeled in (a).

Figure 2: Simulation results for the magnetization reversal of two-segmented cylindrical nanowires with different lengths. The applied field sweeps from 0 to a negative value. The positive $\mathrm{z}$ component of the magnetization (red) points to the left and the negative (blue) one to the right. See supplementary movie M1 for an animated "evolution" of the reversal.

supplementary movie M1).

Lastly, the remanence state of the $50 \mathrm{~nm}$ segments has zero magnetization 


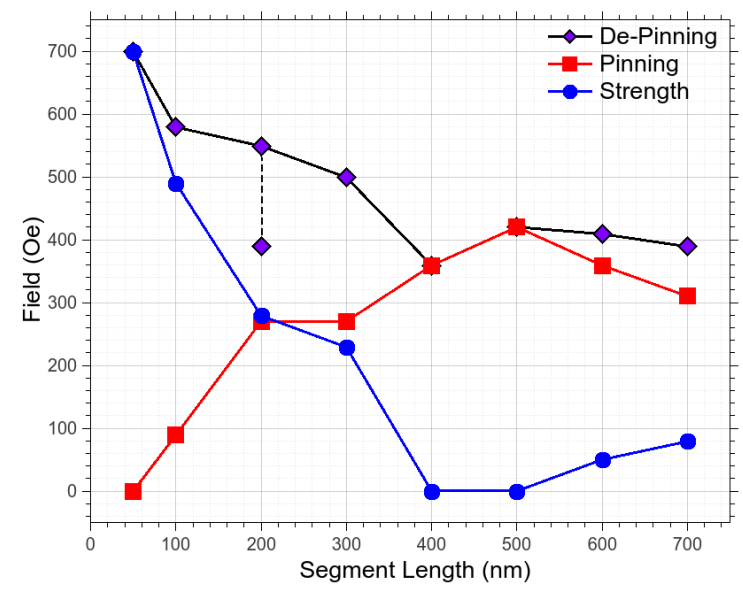

Figure 3: Field values of pinning, depinning and depinning strength for the simulated nanowires. For the $200 \mathrm{~nm}$ segment length, both the first (dashed) and the second (continuous) depinning field values are included (See Fig. 2a.

along $\mathrm{z}$, which is unique compared to the other lengths. This zero magnetization is achieved by a curling magnetization along the $\mathrm{z}$ axis. When increasing the field the magnetization changes by rotation. The sudden jump in magnetization seen around -700 Oe in Fig. $2 \mathrm{a}$ is a transition from curling state to a core-shell structure with the core pointing in the applied field direction.

\subsection{Domain wall position}

For shorter length segments the simulation results show that the domain wall position is right at the interface, as seen in Fig.4. Fig. 4 4 shows the magnetization inside of the CNWs with 100, 200 and $300 \mathrm{~nm}$ long segments, sliced in half along the long axis for a magnetic state in the plateau in Fig. 2a, i.e., with a pinned domain wall. For a $\mathrm{CNW}$ of $80 \mathrm{~nm}$ in diameter and $\sim 700 \mathrm{~nm}$ segment length, the domain wall pins around $150 \mathrm{~nm}$ away from the interface, inside of the $\mathrm{Ni}$ segment (Fig. 2b), which confirms previously reported experimental results [6]. In Fig. $2 \mathrm{~b}$, the $\mathrm{z}$ magnetization component changes direction to the left of the interface, inside the nickel segment. This is attributed to the high stray field of the cobalt, which has a considerable higher saturation magnetization (Table 1) and increases with increased segment length. 


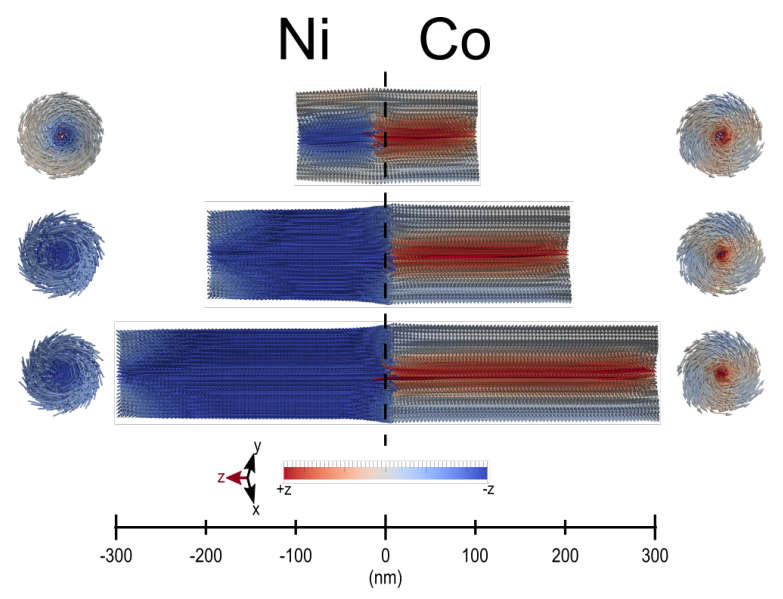

Figure 4: Sliced view of the magnetization components of nanowires of $100 \mathrm{~nm}, 200 \mathrm{~nm}$ and $300 \mathrm{~nm}$ segment length with a pinned domain wall at the interface (vertical dashed line). Slice goes through the center of the nanowire.

\section{Conclusions}

Multisegmented CNWs are attractive materials for novel memory devices. An important step forward is understanding of the relation between domain wall pinning at the interfaces and segment length, whereby the later plays a crucial role in the memory density. Micromagnetic simulations have been carried out on CNWs with $80 \mathrm{~nm}$ in diameter and made of Co and Ni segments, where the material interface acts as pinning site for the domain wall. The conditions of the simulations and their agreement with experimental reports suggest that the exchange interaction at the $\mathrm{Ni} / \mathrm{Co}$ interface plays a minor role in determining the pinning field and strength of the domain wall compared to the cobalt stray field. Several segment lengths were investigated, revealing length-dependent switching mechanisms. They arise from the vortex states at the ends of the nanowires (rods at this length), which are shorter, comparable or longer in length than the length of the segment. In case of the shorter segments (100, 200 and $300 \mathrm{~nm}$ ), the switching mechanism is a combination of vortex state creation and coherent rotation. For intermediate segment lengths (400 and $500 \mathrm{~nm}$ ), the open vortex length at the nickel end is similar to the segments' 

King Abdullah University of Science and Technology (KAUST).

\section{References}

\section{References}

[1] S. S. P. Parkin, M. Hayashi, L. Thomas, Magnetic domain-wall race205

$\mathrm{nm})$, domain wall nucleation and propagation switches the magnetization. The

$1126 /$ science.1145799.

URL http://science.sciencemag.org/content/320/5873/190

[2] H. Mohammed, J. A. Moreno, J. Kosel, Advanced fabrication and characterization of magnetic nanowires, in: Magnetism and Magnetic Materials, IntechOpen, 2017.

[3] E. Berganza, C. Bran, M. Jaafar, M. Vázquez, A. Asenjo, Domain wall pinning in fecocu bamboo-like nanowires, Scientific reports 6 (2016) 29702.

[4] H. Mohammed, E. V. Vidal, Y. P. Ivanov, J. Kosel, Magnetotransport 215 
cylindrical nanowires, IEEE Transactions on Magnetics 52 (7) (2016) 1-5. doi:10.1109/TMAG.2016.2536644

[5] H. Mohammed, H. Corte-Len, Y. P. Ivanov, J. A. Moreno, O. Kazakova, J. Kosel, Angular magnetoresistance of nanowires with alternating cobalt and nickel segments, IEEE Transactions on Magnetics 53 (11) (2017) 1-5. doi:10.1109/TMAG.2017.2718623.

[6] Y. P. Ivanov, A. Chuvilin, S. Lopatin, J. Kosel, Modulated magnetic n nanowires for controlling domain wall motion: Toward 3d magnetic memo-

1 ries, ACS Nano 10 (5) (2016) 5326-5332, pMID: 27138460. arXiv:https: //doi.org/10.1021/acsnano.6b01337, doi:10.1021/acsnano.6b01337 URL https://doi.org/10.1021/acsnano.6b01337

[7] S. Lopatin, Y. P. Ivanov, J. Kosel, A. Chuvilin, Periodic magnetization pattern for controlled domain wall motion in nanowires, Microscopy and Microanalysis 22 (2016) 1678.

[8] S. Lopatin, Y. P. Ivanov, J. Kosel, A. Chuvilin, In-situ study of domain walls propagation and pinning in modulated magnetic nanowires., Microscopy and Microanalysis 22 (2016) 832.

¿ [9] Y. P. Ivanov, A. Chuvilin, S. Lopatin, H. Mohammed, J. Kosel, Di- rect observation of current-induced motion of a $3 \mathrm{~d}$ vortex domain wall 235 in cylindrical nanowires, ACS Applied Materials \& Interfaces 9 (20)

1. (2017) 16741-16744, pMID: 28481499.arXiv:https://doi.org/10.1021/ acsami.7b03404, doi:10.1021/acsami.7b03404.

URL https://doi.org/10.1021/acsami.7b03404

[10] W. Scholz, J. Fidler, T. Schrefl, D. Suess, R. Dittrich, H. Forster, 240 口 V. Tsiantos, Scalable parallel micromagnetic solvers for magnetic nanostructures, Computational Materials Science 28 (2) (2003) 366 - 383, proceedings of the Symposium on Software Development for Process and Materials Design. doi:https://doi.org/10.1016/S0927-0256(03)00119-8. 
URL http://www.sciencedirect.com/science/article/pii/

[11] M. Vousden, M.-A. Bisotti, M. Albert, H. Fangohr, Virtual Micromagnetics: A Framework for Accessible and Reproducible Micromagnetic Simulation, ArXiv e-print:arXiv:1609.05135

[12] C. Ferguson, D. MacLaren, S. McVitie, Metastable magnetic domain walls in cylindrical nanowires, Journal of Magnetism and Magnetic Materials 381 (2015) 457 - 462. doi:https://doi.org/10.1016/j.jmmm.2015.01.027.

口 URL http://www.sciencedirect.com/science/article/pii/ S0304885315000384

[13] Y. P. Ivanov, M. Vzquez, O. Chubykalo-Fesenko, Magnetic reversal modes in cylindrical nanowires, Journal of Physics D: Applied Physics 46 (48) (2013) 485001.

URL http://stacks . iop.org/0022-3727/46/i=48/a=485001

[14] R. C. O'handley, Modern magnetic materials: principles and applications, Wiley, 2000.

[15] J. A. Fernandez-Roldan, R. Perez del Real, C. Bran, M. Vazquez,

口 O. Chubykalo-Fesenko, Magnetization pinning in modulated nanowires: from topological protection to the "corkscrew" mechanism, Nanoscale 10 (2018) 5923-5927. doi:10.1039/C8NR00024G

URL http://dx.doi .org/10.1039/C8NR00024G

265 [16] E. Vilanova Vidal, Y. P. Ivanov, H. Mohammed, J. Kosel, A detailed study of magnetization reversal in individual ni nanowires, Applied Physics Letters 106 (3) (2015) 032403. arXiv:https://doi.org/10.1063/1.4906108, doi:10.1063/1.4906108,

URL https://doi.org/10.1063/1.4906108 